\title{
Hermite spectral and pseudospectral methods for nonlinear partial differential equations in multiple dimensions
}

\author{
XU CHENG-LONG ${ }^{1}$ and GUO BEN-YU² \\ ${ }^{1}$ Department of Applied Mathematics, Tongji University, Shanghai, 200092, P.R. China \\ ${ }^{2}$ Department of Mathematics, Shanghai Normal University, Shanghai, 200234, P.R. China \\ E-mail: clxugol@online.sh.cn / byguo@guomai.sh.cn
}

\begin{abstract}
Hermite approximation in multiple dimensions is investigated. As an example, a spectral scheme and a pseudospectral scheme for the Logistic equation are constructed, respectively. The stability and the convergence of the proposed schemes are proved. Numerical results show the high accuracy of this new approach.
\end{abstract}

Mathematical subject classification: $35 \mathrm{~A} 40,65 \mathrm{M} 12,65 \mathrm{M} 70$.

Key words: Hermite approximation, nonlinear partial differential equations, multiple dimensions.

\section{Introduction}

Spectral methods for partial differential equations in unbounded domains have been received more and more attentions recently. Gottlieb and Orszag [1], Maday, Pernaud-Thomas and Vandeven [2], Coulaud, Funaro and Kavian [3], Funaro [4], and Guo and Shen [5] developed the Laguerre spectral method. While Funaro and Kavian [6] provided some numerical algorithms by using Hermite functions. Furthermore, Guo [7] established some approximation results on the Hermite polynomial approximation with applications to partial differential equations. Guo and $\mathrm{Xu}$ [8] studied the Hermite pseudospectral method and obtained good numerical results.

\#531/02. Received: 28/XI/02. 
As we know, most practical problems are set in multiple dimensions. We may set up some artificial boundaries and impose certain artificial boundary conditions, and then use the usual numerical methods to resolve them in bounded subdomains. But it is not easy to derive the exact boundary conditions, and so some additional errors occur usually. In opposite, if we use the Hermite approximation directly in unbounded domain, then the above trouble could be removed. However, so far, there is no results on the Hermite polynomial and interpolation approximations in multiple dimensions. The aim of this paper is to develop the Hermite spectral and pseudospectral approximations to nonlinear partial differential equations in multiple dimensions.

This paper is organized as follows. In Section 2, we establish some results on the Hermite polynomial approximation and Hermite interpolation in multiple dimensions which play important roles in the analysis of the Hermite spectral and pseudospectral methods. As an example, we construct a Hermite spectral scheme for the multiple dimensions Logistic equation and prove the stability and the convergence of the proposed scheme in Section 3. The corresponding pseudospectral scheme is discussed in Section 4. In the final section, we present some numerical results which show the high accuracy of this new approach.

\section{Hermite approximation in multiple dimensions}

In this section, we consider the Hermite approximation in multiple dimensions. Let $\Lambda_{i}=\left\{x_{i} \mid-\infty<x_{i}<\infty\right\}, \Lambda=\Lambda_{1} \times \Lambda_{2} \times \cdots \times \Lambda_{n}, x=$ $\left(x_{1}, x_{2}, \ldots, x_{n}\right),|x|=\left(\sum_{i=1}^{n} x_{i}^{2}\right)^{\frac{1}{2}}$, and $\omega(x)=e^{-|x|^{2}}$. For $1 \leq p \leq \infty$, let
$L_{\omega}^{p}(\Lambda)=\left\{v \mid v\right.$ is measurable and $\left.\|v\|_{L_{\omega}^{p}}<\infty\right\}$

where

$$
\|v\|_{L_{\omega}^{p}}= \begin{cases}\left(\int_{\Lambda}|v(x)|^{p} \omega(x) d x\right)^{\frac{1}{p}}, & 1 \leq p<\infty, \\ \operatorname{ess} \sup _{x \in \Lambda}|v(x)|, & p=\infty .\end{cases}
$$

In particular, $L_{\omega}^{2}(\Lambda)$ is a Hilbert space with the inner product

$$
(u, v)_{L_{\omega}^{2}(\Lambda)}=\int_{\Lambda} u(x) v(x) \omega(x) d x .
$$


Let $k=\left(k_{1}, k_{2}, \ldots k_{n}\right),|k|=\sum_{i=1}^{n} k_{i}, k_{i}$ being any non-negative integers, and

$$
\partial_{x}^{k} v(x)=\frac{\partial^{|k|} v}{\partial_{x_{1}}^{k_{1}} \cdots \partial_{x_{n}}^{k_{n}}}(x)
$$

For any non-negative integer $m$,

$$
H_{\omega}^{m}(\Lambda)=\left\{v\left|\partial_{x}^{k} v \in L_{\omega}^{2}(\Lambda), 0 \leq\right| k \mid \leq m\right\} .
$$

For any real $r>0$, the space $H_{\omega}^{r}(\Lambda)$ with the semi-norm $|v|_{r, \omega}$ and the norm $\|v\|_{r, \omega}$, is defined by space interpolation as in Adams [9].

Let $l=\left(l_{1}, \ldots l_{n}\right), l_{i}$ being any non-negative integers, and $|l|=\sum_{i=1}^{n} l_{i}$. The Hermite polynomial of degree $l$ is of the form

$$
H_{l}(x)=\prod_{i=1}^{n} H_{l_{i}}\left(x_{i}\right)=(-1)^{|l|} e^{|x|^{2}} \partial_{x}^{l}\left(e^{-|x|^{2}}\right) .
$$

The set of Hermite polynomials is the $L_{\omega}^{2}(\Lambda)$-orthogonal system, i.e.,

$$
\int_{\Lambda} H_{l}(x) H_{k}(x) \omega(x) d x=2^{|l|} l !(\pi)^{\frac{n}{2}} \delta_{l, k}
$$

where $l !=\prod_{i=1}^{n} l_{i}$ ! and

$$
\delta_{l, k}= \begin{cases}1, & l=k \\ 0, & l \neq k\end{cases}
$$

For any $v \in L_{\omega}^{2}(\Lambda)$,

$$
v(x)=\sum_{|l|=0}^{\infty} \hat{v}_{l} H_{l}(x)
$$

where

$$
\hat{v}_{l}=\frac{1}{2^{|l|} l !(\pi)^{\frac{n}{2}}} \int_{\Lambda} v(x) H_{l}(x) \omega(x) d x, \quad|l|=0,1, \cdots .
$$


Let $N$ be any positive integer and $\mathcal{P}_{N}$ be the set of all algebraic polynomials of degree at most $N$ in each variable $x_{i}, 1 \leq i \leq n$. The $L_{\omega}^{2}(\Lambda)$-orthogonal projection $P_{N}: L_{\omega}^{2}(\Lambda) \rightarrow \mathcal{P}_{N}$ is a mapping such that for any $v \in L_{\omega}^{2}(\Lambda)$,

$$
\left(v-P_{N} v, \phi\right)_{\omega}=0, \quad \forall \phi \in \mathcal{P}_{N} .
$$

Let $\omega_{i}\left(x_{i}\right)=e^{-x_{i}^{2}}$ and $P_{N, i}$ be the $L_{\omega_{i}}^{2}\left(\Lambda_{i}\right)$-orthogonal projection.

Lemma 2.1 (see Theorem 2.1 of Guo [7]). For any $v \in H_{\omega_{i}}^{r}\left(\Lambda_{i}\right)$ and $0 \leq \mu \leq r$,

$$
\left\|v-P_{N, i} v\right\|_{\mu, \omega_{i}} \leq c N^{\frac{\mu-r}{2}}\|v\|_{r, \omega_{i}} .
$$

We now consider the multiple-dimensional Hermite polynomial approximation.

Theorem 2.1. For any $v \in H_{\omega}^{r}(\Lambda)$ and $0 \leq \mu \leq r$,

$$
\left\|v-P_{N} v\right\|_{\mu, \omega} \leq c N^{\frac{\mu-r}{2}}\|v\|_{r, \omega} .
$$

Proof. By (2.3) of Guo and Xu [8], $P_{N, i} \partial_{x_{j}} v=\partial_{x_{j}} P_{N, i} v$ for $1 \leq i, j \leq N$. Therefore by Lemma 2.1,

$$
\begin{aligned}
\left\|v-P_{N} v\right\|_{\mu, \omega}= & \left\|v-P_{N, 2} \cdots P_{N, n} v\right\|_{\mu, \omega} \\
& +\left\|P_{N, 2} \cdots P_{N, n}\left(v-P_{N, 1} v\right)\right\|_{\mu, \omega} \\
\leq & \cdots \leq c N^{\frac{\mu-r}{2}}\|v\|_{r, \omega} .
\end{aligned}
$$

In practice, we also need the $H_{\omega}^{1}(\Lambda)$-orthogonal projection $P_{N}^{1}: H_{\omega}^{1}(\Lambda) \rightarrow$ $\mathcal{P}_{N}$. It is a mapping such that for any $v \in H_{\omega}^{1}(\Lambda)$,

$$
\left(\nabla\left(v-P_{N} v\right), \nabla \phi\right)_{\omega}=0, \quad \forall \phi \in \mathcal{P}_{N} .
$$

As explained in Guo [7], we can prove that the projection $P_{N}^{1}$ is exactly the same as $P_{N}$.

Next let

$$
X_{i_{1}, \cdots i_{m}}=\frac{1}{x_{i_{1}} \cdots x_{i_{m}}} \prod_{i=1}^{n} x_{i}, \quad 0 \leq m \leq n .
$$


Lemma 2.2. For any $v \in H_{\omega}^{n}(\Lambda)$,

$$
\left\|X_{i_{1}, \cdots i_{m}} \partial_{x_{1}} \cdots \partial_{x_{m}} v\right\|_{\omega} \leq c\|v\|_{n, \omega} .
$$

Proof. We have from integration by parts that for any $i$,

$$
\begin{aligned}
\left\|x_{i} v\right\|_{\omega}^{2} & =\int_{\Lambda} x_{i}^{2} v^{2}(x) \omega(x) d x \\
& =\frac{1}{2} \int_{\Lambda} v^{2}(x) \omega(x) d x+\int_{\Lambda} x_{i} v(x) \partial_{x_{i}} v(x) \omega(x) d x \\
& \leq \frac{1}{2}\|v\|_{\omega}^{2}+\frac{1}{2}\left\|x_{i} v\right\|_{\omega}^{2}+\frac{1}{2}\left\|\partial_{x_{i}} v\right\|_{\omega}^{2} \\
& =\frac{1}{2}\|v\|_{1, \omega}^{2}+\frac{1}{2}\left\|x_{i} v\right\|_{\omega}^{2}
\end{aligned}
$$

whence

$$
\left\|x_{i} v\right\|_{\omega} \leq\|v\|_{1, \omega} .
$$

Next,

$$
\left\|x_{i} x_{m} v\right\|_{\omega} \leq\left\|x_{m} v\right\|_{1, \omega} \leq c\left\|\left(1+x_{m}\right) v\right\|_{\omega}+\sum_{i=1}^{n}\left\|x_{m} \partial_{x_{i}} v\right\|_{\omega} \leq c\|v\|_{2, \omega} .
$$

By induction,

$$
\left\|v \prod_{i=1}^{n} x_{i}\right\|_{\omega} \leq c\|v\|_{n, \omega}
$$

Similarly

$$
\left\|X_{i_{1}, \cdots, i_{m}} \partial_{x_{i_{1}}} \cdots \partial_{x_{i_{m}}} v\right\|_{\omega} \leq c\|v\|_{n, \omega}
$$

Lemma 2.3. For any $v \in H_{\omega}^{n}(\Lambda)$,

$$
|v(x)| \leq c e^{\frac{1}{2}|x|^{2}}\|v\|_{\omega}^{\frac{1}{2}}\|v\|_{n, \omega}^{\frac{1}{2}} .
$$


Proof. We have

$$
\partial_{x_{i}}\left(e^{-|x|^{2}} v^{2}(x)\right)=-2 x_{i} e^{-|x|^{2}} v^{2}(x)+2 e^{-|x|^{2}} v(x) \partial_{x_{i}} v(x) .
$$

By induction,

$$
\begin{aligned}
\partial_{x_{1}} \cdots \partial_{x_{n}}\left(e^{-|x|^{2}} v^{2}(x)\right)= & (-2)^{n} \prod_{i=1}^{n} x_{i} e^{-|x|^{2}} v^{2}(x) \\
& +c_{1} \sum_{i=1}^{n} X_{i} e^{-|x|^{2}} v(x) \partial_{x_{i}} v(x) \\
& +c_{2} \sum_{1 \leq i_{1}, i_{2} \leq n} X_{i_{1}, i_{2}} e^{-x^{2}} v(x) \partial_{x_{i}} \partial_{x_{j}} v(x) \\
& +\cdots+2 e^{-|x|^{2}} v(x) \partial_{x_{1}} \partial_{x_{2}} \cdots \partial_{x_{n}} v(x)
\end{aligned}
$$

where $c_{i}$ are certain constants. Furthermore let $y=\left(y_{1}, \cdots, y_{n}\right)$. Then

$$
e^{-|x|^{2}} v^{2}(x)=\int_{-\infty}^{x_{1}} \cdots \int_{-\infty}^{x_{n}} \partial_{x_{1}} \partial_{x_{2}} \cdots \partial_{x_{n}}\left(e^{-|y|^{2}} v^{2}(y)\right) d y .
$$

By virtue of (2.1), Lemma 2.2 and the Cauchy inequality,

$$
e^{-|x|^{2}} v^{2}(x) \leq c\|v\|_{\omega}\|v\|_{n, \omega} .
$$

Theorem 2.2. For any $v \in H_{\omega}^{r}(\Lambda)$ and $r \geq n$,

$$
\left\|e^{-\frac{1}{2}|x|^{2}}\left(v-P_{N} v\right)\right\|_{L^{\infty}(\Lambda)} \leq c N^{\frac{n}{4}-\frac{r}{2}}\|v\|_{r, \omega} .
$$

Proof. By Lemma 2.3 and Theorem 2.1, we verify that

$$
\begin{aligned}
\left|v(x)-P_{N} v(x)\right| & \leq c e^{\frac{1}{2}|x|^{2}}\left\|v-P_{N} v\right\|_{\omega}^{\frac{1}{2}}\left\|v-P_{N} v\right\|_{n, \omega}^{\frac{1}{2}} \\
& \leq c e^{\frac{1}{2}|x|^{2}} N^{\frac{n}{4}-\frac{r}{2}}\|v\|_{r, \omega} .
\end{aligned}
$$

This completes the proof.

We now turn to the Hermite-Gauss interpolation. Let $j=\left(j_{1}, \cdots, j_{n}\right), 0 \leq$ $j_{i} \leq N, 1 \leq i \leq n$, and $\sigma_{j_{i}}$ be the zeros of the Hermite polynomial $H_{N+1}\left(x_{i}\right)$. Let

$$
\sigma_{j}=\left(\sigma_{j 1}, \sigma_{j_{2}}, \cdots, \sigma_{j_{n}}\right),
$$


and $\Lambda_{N}$ be the set of all points $\sigma_{j}$. For any $v \in C(\Lambda)$, the Hermite-Gauss interpolant $I_{N} v \in \mathcal{P}_{N}$ is determined by

$$
I_{N} v(x)=v(x), \quad x \in \Lambda_{N} .
$$

Next let $\omega^{(j)}$ be the Christoffel number with respect to $\omega(x)$, namely,

$$
\omega^{(j)}=\prod_{i=1}^{n} \omega^{\left(j_{i}\right)}
$$

where $\omega^{\left(j_{i}\right)}$ are the Christoffel numbers with respect to $\omega_{i}\left(x_{i}\right), 1 \leq i \leq n$.

We introduce the following discrete inner product and norm,

$$
\begin{gathered}
(u, v)_{\omega, N}=\sum_{0 \leq j_{1}, \cdots, j_{n} \leq N} \omega^{\left(j_{1}\right)} \omega^{\left(j_{2}\right)} \cdots \omega^{\left(j_{n}\right)} u\left(\sigma_{j_{1}}, \cdots, \sigma_{j_{n}}\right) v\left(\sigma_{j_{1}}, \cdots, \sigma_{j_{n}}\right), \\
\|v\|_{\omega, N}=(v, v)_{\omega, N}^{\frac{1}{2}} .
\end{gathered}
$$

Clearly

$$
\left(v-I_{N} v, \phi\right)_{\omega, N}=0, \quad \forall \phi \in \mathcal{P}_{N} .
$$

For technical reasons, let

$$
(u, v)_{\omega_{i}, N}=\sum_{0 \leq j_{i} \leq N} \omega^{\left(j_{i}\right)} u\left(\sigma_{j_{i}}\right) v\left(\sigma_{j_{i}}\right), \quad\|v\|_{\omega_{i}, N}=(v, v)_{\omega_{i}, N}^{\frac{1}{2}} .
$$

By Guo and $\mathrm{Xu}$ [8], if $\phi \psi$ is a polynomial on $\Lambda_{i}$ of degree at most $2 N+1$, then

$$
\int_{\Lambda_{i}} \phi\left(x_{i}\right) \psi\left(x_{i}\right) \omega_{i}\left(x_{i}\right) d x_{i}=(\phi, \psi)_{\omega_{i}, N} .
$$

Guo and $\mathrm{Xu}[8]$ also proved that for any $v \in H_{\omega_{i}}^{1}\left(\Lambda_{i}\right)$,

$$
\|v\|_{\omega_{i}, N} \leq c N^{\frac{1}{3}}\|v\|_{\omega_{i}}+c N^{-\frac{1}{6}}\|v\|_{1, \omega_{i}} .
$$

By using (2.3), it can be shown that for any $\phi \psi \in \mathcal{P}_{2 N+1}$,

$$
\int_{\Lambda} \phi(x) \psi(x) \omega(x) d x=(\phi, \psi)_{\omega, N} .
$$

In particular,

$$
\|\phi\|_{\omega}=\|\phi\|_{\omega, N} \quad \forall \phi \in \mathcal{P}_{N} .
$$


Lemma 2.4. For any $v \in H_{\omega}^{n}(\Lambda)$,

$$
\|v\|_{\omega, N} \leq c \sum_{k=0}^{n} N^{\frac{n}{3}-\frac{k}{2}}\|v\|_{k, \omega} .
$$

Proof. We use induction. When $n=1$, the desired result is exactly the same as (2.4). Suppose that the result is valid for $n=m$. Now let $n=m+1$, and $\omega_{m}(x)=e^{-\left(x_{1}^{2}+\cdots+x_{m}^{2}\right)}$. By virtue of (2.4), we have that

$$
\begin{aligned}
& \|v\|_{\omega_{m+1}, N}^{2}=\sum_{0 \leq j_{1}, \cdots j_{m}, j_{m+1} \leq N} \omega^{\left(j_{1}\right)} \cdots \omega^{\left(j_{m}\right)} \omega^{\left(j_{m+1}\right)} v^{2}\left(\sigma_{j_{1}}, \cdots, \sigma_{j_{m}}, \sigma_{j_{m+1}}\right) \\
& =\sum_{0 \leq j_{m+1} \leq N} \omega^{\left(j_{m+1}\right)} \sum_{0 \leq j_{1}, \cdots j_{m} \leq N} \omega^{\left(j_{1}\right)}, \cdots, \omega^{\left(j_{m}\right)} v^{2}\left(\sigma_{j_{1}}, \cdots \sigma_{j_{m}}, \sigma_{j_{m+1}}\right) \\
& \leq \sum_{0 \leq j_{m+1} \leq N} \omega^{\left(j_{m+1}\right)} \sum_{k=0}^{m} c N^{\frac{2 m}{3}-k}\left\|v\left(., \sigma_{j_{m+1}}\right)\right\|_{k, \omega_{m}}^{2} \\
& =c \sum_{k=0}^{m} N^{\frac{2 m}{3}-k} \sum_{0 \leq j_{m+1} \leq N} \omega^{\left(j_{m+1}\right)} \int_{\Lambda_{1} \cdots \Lambda_{m}} e^{-\left(x_{1}^{2}+\cdots+x_{m}^{2}\right)} \\
& \sum_{\substack{0 \leq l_{1}+\cdots+l_{m} \leq k \\
l_{i} \geq 0}}\left(\partial_{x_{1}}^{l_{1}} \cdots \partial_{x_{m}}^{l_{m}} v\left(x_{1}, \cdots, x_{m}, \sigma_{j m+1}\right)\right)^{2} d x_{1} \cdots d x_{m} \\
& =c \sum_{k=0}^{m} N^{\frac{2 m}{3}-k} \int_{\Lambda_{1} \cdots \Lambda_{m}} e^{-\left(x_{1}^{2}+\cdots+x_{m}^{2}\right)} \sum_{\substack{0 \leq l_{1}+\cdots+l_{m} \leq k \\
l_{i} \geq 0}} \\
& \sum_{0 \leq j_{m+1} \leq N} \omega^{\left(j_{m+1}\right)}\left(\partial_{x_{1}}^{l_{1}} \cdots \partial_{x_{m}}^{l_{m}} v\left(x_{1}, \cdots, x_{m}, \sigma_{j_{m+1}}\right)\right)^{2} d x_{1} \cdots d x_{m} \\
& \leq c \sum_{k=0}^{m} N^{\frac{2 m}{3}-k} \int_{\Lambda_{1} \cdots \Lambda_{m}} e^{-\left(x_{1}^{2}+\cdots+x_{m}^{2}\right)}
\end{aligned}
$$

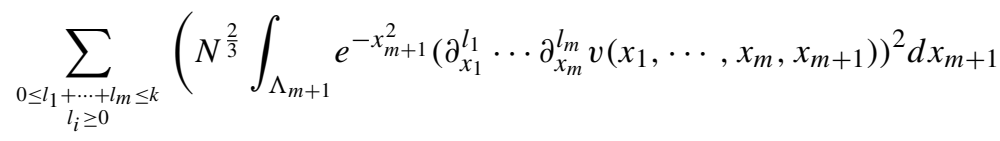

$$
\begin{aligned}
& \left.+N^{-\frac{1}{3}} \int_{\Lambda_{m+1}} e^{-x_{m+1}^{2}}\left(\partial_{x_{m+1}} \partial_{x_{1}}^{l_{1}} \cdots \partial_{x_{m}}^{l_{m}} v\left(x_{1}, \cdots, x_{m}, x_{m+1}\right)\right)^{2} d x_{m+1}\right) d x_{1} \cdots d x_{m} \\
& =c \sum_{k=0}^{m} N^{\frac{2 m}{3}-k} \sum_{\substack{0 \leq l_{1}+\cdots+l_{m} \leq k \\
l_{i} \geq 0}}\left(N^{\frac{2}{3}} \int_{\Lambda} e^{-|x|^{2}}\left(\partial_{x_{1}}^{l_{1}} \cdots \partial_{x_{m}}^{l_{m}} v\left(x_{1}, \cdots, x_{m+1}\right)\right)^{2} d x\right. \\
& \left.+N^{-\frac{1}{3}} \int_{\Lambda} e^{-|x|^{2}}\left(\partial_{x_{m+1}} \partial_{x_{1}}^{l_{1}} \cdots \partial_{x_{m}}^{l_{m}} v\left(x_{1}, \cdots, x_{m+1}\right)\right)^{2} d x\right)
\end{aligned}
$$




$$
\begin{aligned}
& \leq c \sum_{k=0}^{m} N^{\frac{2 m}{3}-k}\left(N^{\frac{2}{3}}\|v\|_{k, \omega_{m+1}}^{2}+N^{-\frac{1}{3}}\|v\|_{k+1, \omega_{m+1}}^{2}\right) \\
& =c \sum_{k=0}^{m} N^{\frac{2 m+2}{3}-k}\|v\|_{k, \omega_{m+1}}^{2}+c \sum_{k=1}^{m+1} N^{\frac{2 m+2}{3}-k}\|v\|_{k, \omega_{m+1}}^{2} \\
& \leq c \sum_{k=0}^{m+1} N^{\frac{2(m+1)}{3}-k}\|v\|_{k, \omega_{m+1}}^{2} .
\end{aligned}
$$

So

$$
\|v\|_{\omega_{m+1}, N} \leq c \sum_{k=0}^{m+1} N^{\frac{m+1}{3}-\frac{k}{2}}\|v\|_{k, \omega_{m+1}}
$$

The induction is compete.

Theorem 2.3. For any $v \in H_{\omega}^{r}(\Lambda), 0 \leq \mu \leq r$ and $r \geq n$,

$$
\left\|v-I_{N} v\right\|_{\mu, \omega} \leq c N^{\frac{n}{3}+\frac{\mu}{2}-\frac{r}{2}}\|v\|_{r, \omega} .
$$

Proof. By Guo [10], for any $\phi \in \mathcal{P}_{N}$ and $\mu \geq 0$,

$$
\|\phi\|_{\mu, \omega} \leq c N^{\frac{\mu}{2}}\|\phi\|_{\omega} .
$$

We have from (2.5), (2.6) and Lemma 2.4 that

$$
\begin{aligned}
\left\|P_{N} v-I_{N} v\right\|_{\mu, \omega} & \leq c N^{\frac{\mu}{2}}\left\|P_{N} v-I_{N} v\right\|_{\omega}=c N^{\frac{\mu}{2}}\left\|I_{N}\left(v-P_{N} v\right)\right\|_{\omega} \\
& =c N^{\frac{\mu}{2}}\left\|v-P_{N} v\right\|_{\omega, N} \\
& \leq c \sum_{k=0}^{n} N^{\frac{n}{3}-\frac{k}{2}+\frac{\mu}{2}}\left\|v-P_{N} v\right\|_{k, \omega} .
\end{aligned}
$$

Therefore by Theorem 2.1,

$$
\begin{aligned}
\left\|v-I_{N} v\right\|_{\mu, \omega} & \leq\left\|v-P_{N} v\right\|_{\mu, \omega}+\left\|P_{N} v-I_{N} v\right\|_{\mu, \omega} \\
& \leq c N^{\frac{n}{3}+\frac{\mu}{2}-\frac{r}{2}}\|v\|_{r, \omega} .
\end{aligned}
$$

Theorem 2.4. For any $v \in H_{\omega}^{r}(\Lambda)$ and $r \geq n$,

$$
\left\|\left(v-I_{N} v\right) e^{-\frac{1}{2}|x|^{2}}\right\|_{L^{\infty}(\Lambda)} \leq c N^{\frac{7 n}{12}-\frac{r}{2}}\|v\|_{r, \omega} .
$$


Proof. Thanks to Lemma 2.3 and Theorem 2.3, we get that for any $x \in \Lambda$,

$$
\begin{aligned}
\left|v(x)-I_{N} v(x)\right| & \leq c e^{\frac{1}{2}|x|^{2}}\left\|v-I_{N} v\right\|_{\omega}^{\frac{1}{2}}\left\|v-I_{N} v\right\|_{n, \omega}^{\frac{1}{2}} \\
& \leq c e^{\frac{1}{2}|x|^{2}} N N^{\frac{7 n}{12}-\frac{r}{2}}\|v\|_{r, \omega} .
\end{aligned}
$$

The desired result follows.

We have from (2.5) and Theorem 2.3 that for any $v \in H_{\omega}^{r}(\Lambda), \phi \in \mathcal{P}_{N}$ and $r \geq n$,

$$
\begin{aligned}
\left|(v, \phi)_{\omega}-(v, \phi)_{\omega, N}\right| & =\left|\left(v-I_{N} v, \phi\right)_{\omega}\right| \leq c\left\|v-I_{N} v\right\|_{\omega}\|\phi\|_{\omega} \\
& \leq c N^{\frac{n}{3}-\frac{r}{2}}\|v\|_{r, \omega}\|\phi\| .
\end{aligned}
$$

\section{Hermite spectral scheme for the logistic model}

This section is for application of the Hermite spectral approximation to the Logistic equation in two-dimensions. We construct a Hermite spectral scheme, and prove its stability and convergence. The main idea and techniques in this section are also applicable to other nonlinear partial differential equations in $n$-dimensions.

Let $y=\left(y_{1}, y_{2}\right)$ and $\Lambda=\left(\Lambda_{1}, \Lambda_{2}\right) . V(y, s)$ describes the population of budworm. $g(y, s)$ and $V_{0}(y)$ are the source term and the initial state of population, respectively. Then the Logistic model takes the form

$$
\begin{cases}\partial_{s} V-\partial_{y_{1}}^{2} V-\partial_{y_{2}}^{2} V=V(1-V)+g, & y \in \Lambda, 0<s \leq T, \\ V(y, 0)=V_{0}(y), & y \in \Lambda .\end{cases}
$$

As pointed out in [7], the Laplacian in (3.1) does not correspond to a positivedefinite bilinear form in $H_{\omega}^{1}(\Lambda)$, and so (3.1) is not well-posed in the weighted space. So we take the following similarity transformation

$$
x=\left(x_{1}, x_{2}\right), \quad x_{i}=\frac{y_{i}}{2 \sqrt{1+s}}, i=1,2, t=\ln (1+s) .
$$

Let

$$
W(x, t)=V\left(2 x e^{\frac{t}{2}}, e^{t}-1\right), \quad W_{0}(x)=V_{0}(2 x), \quad \tilde{g}(x, t)=g\left(2 x e^{\frac{t}{2}}, e^{t}-1\right) .
$$

Then

$$
\partial_{s} V=e^{-t}\left(\partial_{t} W-\frac{1}{2} x_{1} \partial_{x_{1}} W-\frac{1}{2} x_{2} \partial_{x_{2}} W\right), \quad \partial_{y_{i}}^{2} V=\frac{1}{4} e^{-t} \partial_{x_{i}}^{2} W, i=1,2 .
$$


So (3.1) becomes

$$
\left\{\begin{array}{cc}
\partial_{t} W-\frac{1}{2} x_{1} \partial_{x_{1}} W-\frac{1}{2} x_{2} \partial_{x_{2}} W-\frac{1}{4} \partial_{x_{1}}^{2} W-\frac{1}{4} \partial_{x_{2}}^{2} W \\
=e^{t} W(1-W)+e^{t} \tilde{g}, & x \in \Lambda, 0<t \leq \ln (1+T), \\
W(x, 0)=W_{0}(x), & x \in \Lambda .
\end{array}\right.
$$

Further, let

$$
U=e^{|x|^{2}} W, \quad U_{0}(x)=e^{|x|^{2}} W_{0}(x), \quad f=e^{t+|x|^{2}} \tilde{g} .
$$

Then problem (3.3) is changed into

$$
\left\{\begin{array}{cl}
\partial_{t} U+\frac{1}{2} U+\frac{1}{2} x_{1} \partial_{x_{1}} U+\frac{1}{2} x_{2} \partial_{x_{2}} U-\frac{1}{4} \partial_{x_{1}}^{2} U-\frac{1}{4} \partial_{x_{2}}^{2} U \\
=e^{t} U\left(1-e^{-|x|^{2}} U\right)+f, & x \in \Lambda, 0<t \leq \ln (1+T), \\
U(x, 0)=U_{0}(x), & x \in \Lambda .
\end{array}\right.
$$

The weak formulation of (3.4) is to find $U \in L^{2}\left(0, \ln (1+T) ; H_{\omega}^{1}(\Lambda)\right) \cap$ $L^{\infty}\left(0, \ln (1+T) ; L_{\omega}^{2}(\Lambda)\right)$ such that

$$
\left\{\begin{array}{c}
\left(\partial_{t} U(t), v\right)_{\omega}+\frac{1}{2}(U(t), v)_{\omega}+\frac{1}{4}(\nabla U(t), \nabla v)_{\omega} \\
=e^{t}\left(U(t)-e^{-|x|^{2}} U^{2}(t), v\right)_{\omega}+(f(t), v)_{\omega}, \\
\forall v \in H_{\omega}^{1}(\Lambda), 0<t \leq \ln (1+T), \\
U(0)=U_{0} .
\end{array}\right.
$$

The Hermite spectral scheme for (3.5) is to find $u_{N}(t) \in \mathcal{P}_{N}$ for all $0<t \leq$ $\ln (1+T)$, such that

$$
\left\{\begin{array}{c}
\left(\partial_{t} u_{N}(t), \phi\right)_{\omega}+\frac{1}{2}\left(u_{N}(t), \phi\right)_{\omega}+\frac{1}{4}\left(\nabla u_{N}(t), \nabla \phi\right)_{\omega} \\
=e^{t}\left(u_{N}(t)-e^{-|x|^{2}} u_{N}^{2}(t), \phi\right)_{\omega}+(f(t), \phi)_{\omega}, \\
\forall \phi \in \mathcal{P}_{N}, 0<t \leq \ln (1+T) \\
u_{N}(0)=u_{N, 0}=P_{N} U_{0} .
\end{array}\right.
$$

We give some Lemmas which will be used in the analysis of the stability and the convergence of scheme (3.6). 
Lemma 3.1 (see Lemma 2.3 of Guo [7]). For any $v \in H_{\omega_{i}}^{1}\left(\Lambda_{i}\right)$,

$$
\left\|x_{i} v\right\|_{\omega_{i}} \leq\|v\|_{1, \omega_{i}} .
$$

Lemma 3.2. For any $v \in H_{\omega_{i}}^{1}\left(\Lambda_{i}\right)$,

$$
\left\|\partial_{x_{i}}\left(e^{-\frac{x_{i}^{2}}{2}} v\right)\right\| \leq \sqrt{2}\|v\|_{1, \omega_{i}} .
$$

Proof. By integration by parts and Lemma 3.1, we obtain that

$$
\begin{aligned}
& \int_{\Lambda_{i}}\left(\partial_{x_{i}}\left(e^{-\frac{x_{i}^{2}}{2}} v\left(x_{i}\right)\right)\right)^{2} d x_{i} \\
& \quad=\int_{\Lambda_{i}} e^{-x_{i}^{2}}\left(x_{i}^{2} v^{2}\left(x_{i}\right)-2 x_{i} v\left(x_{i}\right) \partial_{x_{i}} v\left(x_{i}\right)+\left(\partial_{x_{i}} v\left(x_{i}\right)\right)^{2}\right) d x_{i} \\
& \quad=\frac{1}{2} \int_{\Lambda_{i}} e^{-x_{i}^{2}} v^{2}\left(x_{i}\right) d x_{i}-\int_{\Lambda_{i}} x_{i} e^{-x_{i}^{2}} v\left(x_{i}\right) \partial_{x_{i}} v\left(x_{i}\right) d x_{i}+\int_{\Lambda_{i}} e^{-x_{i}^{2}}\left(\partial_{x_{i}} v\left(x_{i}\right)\right)^{2} d x_{i} \\
& \quad \leq \frac{1}{2}\|v\|_{\omega_{i}}^{2}+\left\|x_{i} v\right\|_{\omega_{i}}\left\|\partial_{x_{i}} v\right\|_{\omega_{i}}+\left\|\partial_{x_{i}} v\right\|_{\omega_{i}}^{2} \leq 2\|v\|_{1, \omega_{i}}^{2} .
\end{aligned}
$$

Lemma 3.3. For any $v \in H_{\omega}^{1}(\Lambda)$,

$$
\int_{\Lambda} e^{-2|x|^{2}} v^{4}(x) d x \leq 8\|v\|_{\omega}^{2}\|v\|_{1, \omega}^{2}
$$

Proof. For any $x \in \Lambda$,

$$
\begin{aligned}
e^{-\left(x_{1}^{2}+x_{2}^{2}\right)} v^{2}\left(x_{1}, x_{2}\right) & =2 \int_{-\infty}^{x_{1}} e^{-\frac{\xi^{2}+x_{2}^{2}}{2}} v\left(\xi, x_{2}\right) \partial_{\xi}\left(e^{-\frac{\xi^{2}+x_{2}^{2}}{2}} v\left(\xi, x_{2}\right)\right) d \xi \\
& \leq 2 \int_{-\infty}^{\infty} e^{-\frac{\xi^{2}+x_{2}^{2}}{2}}\left|v\left(\xi, x_{2}\right)\right|\left|\partial_{\xi}\left(e^{-\frac{\xi^{2}+x_{2}^{2}}{2}} v\left(\xi, x_{2}\right)\right)\right| d \xi
\end{aligned}
$$

Similarly

$$
e^{-\left(x_{1}^{2}+x_{2}^{2}\right)} v^{2}\left(x_{1}, x_{2}\right) \leq 2 \int_{-\infty}^{\infty} e^{-\frac{x_{1}^{2}+\eta^{2}}{2}}\left|v\left(x_{1}, \eta\right)\right|\left|\partial_{\eta}\left(e^{-\frac{x_{1}^{2}+\eta^{2}}{2}} v\left(x_{1}, \eta\right)\right)\right| d \eta .
$$


Thus we have

$$
\begin{aligned}
e^{-2\left(x_{1}^{2}+x_{2}^{2}\right)} v^{4}\left(x_{1}, x_{2}\right) \leq & 4 \int_{-\infty}^{\infty} e^{-\frac{\xi^{2}+x_{2}^{2}}{2}}\left|v\left(\xi, x_{2}\right)\right|\left|\partial_{\xi}\left(e^{-\frac{\xi^{2}+x_{2}^{2}}{2}} v\left(\xi, x_{2}\right)\right)\right| d \xi \\
& \times \int_{-\infty}^{\infty} e^{-\frac{x_{1}^{2}+\eta^{2}}{2}}\left|v\left(x_{1}, \eta\right)\right|\left|\partial_{\eta}\left(e^{-\frac{x_{1}^{2}+\eta^{2}}{2}} v\left(x_{1}, \eta\right)\right)\right| d \eta
\end{aligned}
$$

The above with Lemma 3.2 leads to

$$
\begin{aligned}
\int_{\Lambda} & e^{-2|x|^{2}} v^{4}(x) d x \\
\leq & 4 \int_{\Lambda} e^{-\frac{\xi^{2}+x_{2}^{2}}{2}}\left|v\left(\xi, x_{2}\right)\right|\left|\partial_{\xi}\left(e^{-\frac{\xi^{2}+x_{2}^{2}}{2}} v\left(\xi, x_{2}\right)\right)\right| d \xi d x_{2} \\
& \times \int_{\Lambda} e^{-\frac{x_{1}^{2}+\eta^{2}}{2}}\left|v\left(x_{1}, \eta\right)\right|\left|\partial_{\eta}\left(e^{-\frac{x_{1}^{2}+\eta^{2}}{2}} v\left(x_{1}, \eta\right)\right)\right| d x_{1} d \eta \\
\leq & 4\left(\int_{\Lambda} e^{-\left(\xi^{2}+x_{2}^{2}\right)} v^{2}\left(\xi, x_{2}\right) d \xi d x_{2}\right)^{\frac{1}{2}}\left(\int_{\Lambda}\left(\partial_{\xi}\left(e^{-\frac{\xi^{2}+x_{2}^{2}}{2}} v\left(\xi, x_{2}\right)\right)\right)^{2} d \xi d x_{2}\right)^{\frac{1}{2}} \\
& \times\left(\int_{\Lambda} e^{-\left(x_{1}^{2}+\eta^{2}\right)} v^{2}\left(x_{1}, \eta\right) d x_{1} d \eta\right)^{\frac{1}{2}}\left(\int_{\Lambda}\left(\partial_{\eta}\left(e^{-\frac{x_{1}^{2}+\eta^{2}}{2}} v\left(x_{1}, \eta\right)\right)\right)^{2} d x_{1} d \eta\right)^{\frac{1}{2}} \\
\leq & 8\|v\|_{\omega}^{2}\|v\|_{1, \omega}^{2} .
\end{aligned}
$$

We now consider the stability of (3.6). Assume that $f$ and $u_{N, 0}$ have the errors $\tilde{f}$ and $\tilde{u}_{N, 0}$, respectively. They induce the error of numerical solution $u_{N}$, denoted by $\tilde{u}_{N}$. Then the errors fulfill the following equation

$$
\left\{\begin{array}{l}
\left(\partial_{t} \tilde{u}_{N}(t), \phi\right)_{\omega}+\frac{1}{2}\left(\tilde{u}_{N}(t), \phi\right)_{\omega}+\frac{1}{4}\left(\nabla \tilde{u}_{N}(t), \nabla \phi\right)_{\omega} \\
=e^{t}\left(\tilde{u}_{N}(t)-e^{-|x|^{2}}\left(\tilde{u}_{N}^{2}(t)+2 u_{N}(t) \tilde{u}_{N}(t)\right), \phi\right)_{\omega}+(\tilde{f}(t), \phi)_{\omega}, \\
\forall \phi \in \mathcal{P}_{N}, 0<t \leq \ln (1+T), \\
\tilde{u}_{N}(0)=\tilde{u}_{N, 0} .
\end{array}\right.
$$


By taking $\phi=2 \tilde{u}_{N}$ in (3.7), we obtain that

$$
\begin{aligned}
\frac{d}{d t}\left\|\tilde{u}_{N}(t)\right\|_{\omega}^{2}+\frac{1}{2}\left\|\tilde{u}_{N}(t)\right\|_{1, \omega}^{2} \\
\leq 2 e^{t}\left(\left\|\tilde{u}_{N}(t)\right\|_{\omega}^{2}-\left(e^{-|x|^{2}} \tilde{u}_{N}^{2}(t), \tilde{u}_{N}(t)\right)_{\omega}\right) \\
\quad-2\left(e^{-|x|^{2}} u_{N}(t) \tilde{u}_{N}(t), \tilde{u}_{N}(t)\right)_{\omega}+2\|\tilde{f}(t)\|_{\omega}^{2} .
\end{aligned}
$$

By the Schwartz inequality and Lemma 3.3,

$$
\begin{aligned}
&\left|\left(e^{-|x|^{2}} \tilde{u}_{N}^{2}(t), \tilde{u}_{N}(t)\right)_{\omega}\right| \leq 2 \sqrt{2}\left\|\tilde{u}_{N}(t)\right\|_{\omega}^{2}\left\|\tilde{u}_{N}(t)\right\|_{1, \omega} \\
& \leq 2 \sqrt{2}\left\|\tilde{u}_{N}(t)\right\|_{\omega}\left\|\tilde{u}_{N}(t)\right\|_{1, \omega}^{2}, \\
&\left|\left(e^{-|x|^{2}} u_{N}(t) \tilde{u}_{N}(t), \tilde{u}_{N}(t)\right)_{\omega}\right|=\left|\left(u_{N}(t), e^{-|x|^{2}} \tilde{u}_{N}^{2}(t)\right)_{\omega}\right| \\
& \leq 2 \sqrt{2}\left\|u_{N}(t)\right\|_{\omega}\left\|\tilde{u}_{N}(t)\right\|_{\omega}\left\|\tilde{u}_{N}(t)\right\|_{1, \omega} \\
& \leq \frac{e^{-t}}{16}\left\|\tilde{u}_{N}(t)\right\|_{1, \omega}^{2}+32 e^{t}\left\|u_{N}(t)\right\|_{\omega}^{2}\left\|\tilde{u}_{N}(t)\right\|_{\omega}^{2} .
\end{aligned}
$$

Substituting (3.9) and (3.10) into (3.8) and integrating the result with respect to $t$, we obtain that

$$
\begin{gathered}
\left\|\tilde{u}_{N}(t)\right\|_{\omega}^{2}+\int_{0}^{t}\left(\frac{1}{4}-c_{1}(T)\left\|\tilde{u}_{N}(\eta)\right\|_{\omega}\right)\left\|\tilde{u}_{N}(\eta)\right\|_{1, \omega}^{2} d \eta \\
\leq \rho\left(\tilde{u}_{N, 0}, \tilde{f}, t\right)+c_{2}\left(u_{N}, T\right) \int_{0}^{t}\left\|\tilde{u}_{N}(\eta)\right\|_{\omega}^{2} d \eta
\end{gathered}
$$

where

$$
\begin{gathered}
\rho\left(\tilde{u}_{N, 0}, \tilde{f}, t\right)=\left\|\tilde{u}_{N, 0}\right\|_{\omega}^{2}+2 \int_{0}^{t}\|\tilde{f}(\eta)\|_{\omega}^{2} d \eta, \\
c_{1}(T)=4 \sqrt{2}(1+T), \\
c_{2}\left(u_{N}, T\right)=2(1+T)\left(1+64(1+T)\left\|u_{N}\right\|_{L^{\infty}\left(0, \ln (1+T) ; L_{\omega}^{2}(\Lambda)\right)}^{2}\right) .
\end{gathered}
$$

Lemma 3.4 (see Lemma 3.1 of Guo [7]). Assume that

(i) the constants $b_{1}>0, b_{2}>0, b_{3}>0$ and $d \geq 0$,

(ii) $Z(t)$ and $A(t)$ are non-negative functions of $t$, 
(iii) $d \leq \frac{b_{1}^{2}}{b_{2}^{2}} e^{-b_{3} t_{1}}$ for certain $t_{1}>0$,

(iv) for all $t \leq t_{1}$,

$$
Z(t)+\int_{0}^{t}\left(b_{1}-b_{2} Z^{\frac{1}{2}}(\eta)\right) A(\eta) d \eta \leq d+b_{3} \int_{0}^{t} Z(\eta) d \eta .
$$

Then for all $t \leq t_{1}$,

$$
Z(t) \leq d e^{b_{3} t}
$$

Applying Lemma 3.4 to (3.11), we obtain the following result.

Theorem 3.1. Let $0 \leq a<1$ and $u_{N}(t)$ be the solution of (3.6). If for certain $t_{1}>0$,

$$
\rho\left(\tilde{u}_{N, 0}, \tilde{f}_{0}, t_{1}\right) \leq \frac{(1-a)^{2}}{16 c_{1}^{2}(T)} e^{-c_{2}\left(u_{N}, T\right) t_{1}},
$$

then for all $t \leq t_{1}$,

$$
\left\|\tilde{u}_{N}(t)\right\|_{\omega}^{2}+\frac{a}{4} \int_{0}^{t}\left\|\tilde{u}_{N}(\eta)\right\|_{1, \omega}^{2} \mathrm{~d} \eta \leq \rho\left(\tilde{u}_{N, 0}, \tilde{f}_{0}, t\right) e^{c_{2}\left(u_{N}, T\right) t} .
$$

Remark 3.1. Theorem 3.1 indicates that the scheme (3.6) is of generalized stability in the sense of Guo $[11,12]$, and of restricted stability in the sense of Stetter[13]. It means that the computation is stable for small errors of data.

We next deal with the convergence of scheme (3.6). Let $U$ be the solution of (3.5), and $U_{N}=P_{N} U=P_{N}^{1} U$. We get from (3.5) that

$$
\left\{\begin{array}{l}
\left(\partial_{t} U_{N}(t), \phi\right)_{\omega}+\frac{1}{2}\left(U_{N}(t), \phi\right)_{\omega}+\frac{1}{4}\left(\nabla U_{N}(t), \nabla \phi\right)_{\omega} \\
=e^{t}\left(U_{N}(t)-e^{-|x|^{2}} U_{N}^{2}(t), \phi\right)_{\omega}+(f(t), \phi)_{\omega} \\
\quad+G_{1}(t, \phi)+G_{2}(t, \phi), \quad \forall \phi \in \mathcal{P}_{N}, 0<t \leq \ln (1+T), \\
U_{N}(0)=P_{N} U_{0}
\end{array}\right.
$$


where

$$
\begin{gathered}
G_{1}(t, \phi)=\left(\partial_{t} U_{N}(t)-\partial_{t} U(t), \phi\right)_{\omega}, \\
G_{2}(t, \phi)=e^{t}\left(e^{-|x|^{2}}\left(U_{N}^{2}(t)-U^{2}(t)\right), \phi\right)_{\omega} .
\end{gathered}
$$

Let $\tilde{U}_{N}(t)=u_{N}(t)-U_{N}(t)$. By subtracting (3.12) from (3.6), we get that

$$
\left\{\begin{array}{l}
\left(\partial_{t} \tilde{U}_{N}(t), \phi\right)_{\omega}+\frac{1}{2}\left(\tilde{U}_{N}(t), \phi\right)_{\omega}+\frac{1}{4}\left(\nabla \tilde{U}_{N}(t), \nabla \phi\right)_{\omega} \\
=e^{t}\left(\tilde{U}_{N}(t)-e^{-|x|^{2}}\left(\tilde{U}_{N}^{2}(t)+2 U_{N}(t) \tilde{U}_{N}(t)\right), \phi\right)_{\omega} \\
\quad-G_{1}(t, \phi)-G_{2}(t, \phi), \quad \forall \phi \in \mathcal{P}_{N}, 0<t \leq \ln (1+T), \\
\tilde{U}_{N}(0)=0 .
\end{array}\right.
$$

Comparing (3.13) to (3.7), we only need to estimate the terms $\left|G_{i}\left(t, \tilde{U}_{N}(t)\right)\right|$. By Theorem 2.1,

$$
\left|G_{1}\left(t, \tilde{U}_{N}(t)\right)\right| \leq\left\|\tilde{U}_{N}(t)\right\|_{\omega}^{2}+c N^{-r}\left\|\partial_{t} U(t)\right\|_{r, \omega}^{2} .
$$

By Lemma 3.3 and Theorem 2.1, we have that for $r \geq 1$,

$$
\begin{aligned}
\left|G_{2}\left(t, \tilde{U}_{N}(t)\right)\right|= & e^{t}\left|\left(e^{-|x|^{2}}\left(U(t)+U_{N}(t)\right) \tilde{U}_{N}(t), U(t)-U_{N}(t)\right)_{\omega}\right| \\
\leq & \frac{1}{2} c_{1}(T)\left\|U(t)+U_{N}(t)\right\|_{\omega}^{\frac{1}{2}}\left\|U(t)+U_{N}(t)\right\|_{1, \omega}^{\frac{1}{2}} \\
& \left\|\tilde{U}_{N}(t)\right\|_{\omega}^{\frac{1}{2}}\left\|\tilde{U}_{N}(t)\right\|_{1, \omega}^{\frac{1}{2}}\left\|U(t)-U_{N}(t)\right\|_{\omega} \\
\leq & \frac{1}{16}\left\|\tilde{U}_{N}(t)\right\|_{1, \omega}^{2}+c c_{1}^{2}(T) N^{-r}\|U(t)\|_{r, \omega}^{4} .
\end{aligned}
$$

Finally we obtain the following result.

Theorem 3.2. If $U \in H^{1}\left(0, \ln (1+T) ; H_{\omega}^{r}(\Lambda)\right)$ with $r \geq 1$. Then for all $0 \leq t \leq \ln (1+T)$,

$$
\left\|\tilde{U}_{N}(t)\right\|_{\omega}^{2}+\int_{0}^{t}\left\|\tilde{U}_{N}(\eta)\right\|_{1, \omega}^{2} d \eta \leq c^{*} N^{-r}
$$

where $c^{*}$ is a positive constant depending only on $T$ and the norms of $U$ in the spaces mentioned above. 
Remark 3.2. By Theorem 3.2 and Theorem 2.1, we have that under the conditions of Theorem 3.2,

$$
\left\|u_{N}(t)-U(t)\right\|_{\omega}^{2}+N^{-1} \int_{0}^{t}\left\|u_{N}(\eta)-U(\eta)\right\|_{1, \omega}^{2} d \eta \leq c^{*} N^{-r} .
$$

Remark 3.3. Since $c_{2}\left(U_{N}, T\right)$ depends on $T^{2}$ linearly, we can see that $c^{*}$ depends on $T^{3}$ linearly.

\section{Hermite pseudospectral scheme for logistic model}

In this section, we consider a Hermite pseudospectral scheme for (3.5). Let $n=2$, we use the same notations as in Section 2.

The Hermite pseudospectral scheme for (3.5) is to find $u_{N}(t) \in \mathcal{P}_{N}$ for all $0 \leq t \leq \ln (1+T)$, such that

$$
\left\{\begin{array}{c}
\left(\partial_{t} u_{N}(t), \phi\right)_{\omega}+\frac{1}{2}\left(u_{N}(t), \phi\right)_{\omega}+\frac{1}{4}\left(\nabla u_{N}(t), \nabla \phi\right)_{\omega} \\
=e^{t}\left(u_{N}(t)-e^{-|x|^{2}} u_{N}^{2}(t), \phi\right)_{\omega, N}+(f(t), \phi)_{\omega, N}, \\
\forall \phi \in \mathcal{P}_{N}, 0<t \leq \ln (1+T), \\
u_{N}(0)=u_{N, 0}=I_{N} U_{0} .
\end{array}\right.
$$

Remark 4.1. By (2.3), the first formula of (4.1) is equivalent to

$$
\begin{gathered}
\left(\partial_{t} u_{N}(t), \phi\right)_{\omega, N}+\frac{1}{2}\left(u_{N}(t), \phi\right)_{\omega, N}+\frac{1}{4}\left(\nabla u_{N}(t), \nabla \phi\right)_{\omega, N} \\
=e^{t}\left(u_{N}(t)-e^{-|x|^{2}} u_{N}^{2}(t), \phi\right)_{\omega, N}+(f(t), \phi)_{\omega, N}, \\
\forall \phi \in \mathcal{P}_{N}, 0<t \leq \ln (1+T) .
\end{gathered}
$$

The following Lemma will be used in the analysis of the stability and the convergence of scheme (4.1).

Lemma 4.1. For any $v \in \mathcal{P}_{N}$,

$$
\sum_{0 \leq j_{1}, j_{2} \leq N} e^{-\left(\sigma_{j_{1}}^{2}+\sigma_{j_{2}}^{2}\right)} \omega^{(j)} v^{4}\left(\sigma_{j}\right) \leq 8\|v\|_{\omega}^{2}\|v\|_{1, \omega}^{2}
$$


Proof. We have

$$
\begin{aligned}
e^{-\sigma_{j_{1}}^{2}} v^{2}\left(\sigma_{j}\right) & =2 \int_{-\infty}^{\sigma_{j_{1}}} e^{-\frac{x_{1}^{2}}{2}} v\left(x_{1}, \sigma_{j_{2}}\right) \partial_{x_{1}}\left(e^{-\frac{x_{1}^{2}}{2}} v\left(x_{1}, \sigma_{j_{2}}\right)\right) d x_{1} \\
& \leq 2 \int_{\Lambda_{1}} e^{-\frac{x_{1}^{2}}{2}}\left|v\left(x_{1}, \sigma_{j_{2}}\right)\right|\left|\partial_{x_{1}}\left(e^{-\frac{x_{1}^{2}}{2}} v\left(x_{1}, \sigma_{j_{2}}\right)\right)\right| d x_{1} .
\end{aligned}
$$

Similarly

$$
e^{-\sigma_{j_{2}}^{2}} v^{2}\left(\sigma_{j}\right) \leq 2 \int_{\Lambda_{2}} e^{-\frac{x_{2}^{2}}{2}}\left|v\left(\sigma_{j_{1}}, x_{2}\right)\right|\left|\partial_{x_{2}}\left(e^{-\frac{x_{2}^{2}}{2}} v\left(\sigma_{j_{1}}, x_{2}\right)\right)\right| d x_{2} .
$$

Therefore, by the Hölder inequality, (2.4) and Lemma 3.2, we obtain that

$$
\begin{aligned}
\sum_{0 \leq j_{1}, j_{2} \leq N} e^{-\left|\sigma_{j}\right|^{2}} \omega^{(j)} v^{4}\left(\sigma_{j}\right) & \\
\leq & 4 \int_{\Lambda_{1}} \sum_{0 \leq j_{2} \leq N} \omega^{\left(j_{2}\right)} e^{-\frac{x_{1}^{2}}{2}}\left|v\left(x_{1}, \sigma_{j_{2}}\right)\right|\left|\partial_{x_{1}}\left(e^{-\frac{x_{1}^{2}}{2}} v\left(x_{1}, \sigma_{j_{2}}\right)\right)\right| d x_{1} \\
& \times \int_{\Lambda_{2}} \sum_{0 \leq j_{1} \leq N} \omega^{\left(j_{1}\right)} e^{-\frac{x_{2}^{2}}{2}}\left|v\left(\sigma_{j_{1}}, x_{2}\right)\right|\left|\partial_{x_{2}}\left(e^{-\frac{x_{2}^{2}}{2}} v\left(\sigma_{j_{1}}, x_{2}\right)\right)\right| d x_{2} \\
\leq & 4\left(\int_{\Lambda_{1}} \sum_{0 \leq j_{2} \leq N} \omega^{\left(j_{2}\right)} e^{-x_{1}^{2}} v^{2}\left(x_{1}, \sigma_{j_{2}}\right) d x_{1}\right)^{\frac{1}{2}} \\
& \left(\int_{\Lambda_{1}} \sum_{0 \leq j_{2} \leq N} \omega^{\left(j_{2}\right)}\left(\partial_{x_{1}}\left(e^{-\frac{x_{1}^{2}}{2}} v\left(x_{1}, \sigma_{j_{2}}\right)\right)\right)^{2} d x_{1}\right)^{\frac{1}{2}} \\
& \times\left(\int_{\Lambda_{2}} \sum_{0 \leq j_{1} \leq N} \omega^{\left(j_{1}\right)} e^{-x_{2}^{2}} v^{2}\left(\sigma_{j_{1}}, x_{2}\right) d x_{2}\right)^{\frac{1}{2}} \\
& \left(\int_{\Lambda_{2}} \sum_{0 \leq j_{1} \leq N} \omega^{\left(j_{1}\right)}\left(\partial_{x_{2}}\left(e^{-\frac{x_{2}^{2}}{2}} v\left(\sigma_{j_{1}}, x_{2}\right)\right)\right)^{2} d x_{2}\right)^{\frac{1}{2}} \\
= & 4\left(\int_{\Lambda} e^{-|x|^{2}} v^{2}(x) d x\right)^{\frac{1}{2}}\left(\int_{\Lambda} e^{-x_{2}^{2}}\left(\partial_{x_{1}}\left(e^{-\frac{x_{1}^{2}}{2}} v(x)\right)\right)^{2} d x\right)^{\frac{1}{2}} \\
& \times\left(\int_{\Lambda} e^{-|x|^{2}} v^{2}(x) d x\right)^{\frac{1}{2}}\left(\int_{\Lambda} e^{-x_{1}^{2}}\left(\partial_{x_{2}}\left(e^{-\frac{x_{2}^{2}}{2}} v(x)\right)\right)^{2} d x\right)_{\omega}^{\frac{1}{2}}\|v\|_{1, \omega}^{2} .
\end{aligned}
$$


We now analyze the stability of (4.1). Assume that $f$ and $u_{N, 0}$ have the errors $\tilde{f}$ and $\tilde{u}_{N, 0}$, respectively, which induce the error of $u_{N}$, denoted by $\tilde{u}_{N}$. Then the errors fulfill the following equation

$$
\left\{\begin{array}{l}
\left(\partial_{t} \tilde{u}_{N}(t), \phi\right)_{\omega}+\frac{1}{2}\left(\tilde{u}_{N}(t), \phi\right)_{\omega}+\frac{1}{4}\left(\nabla \tilde{u}_{N}(t), \nabla \phi\right)_{\omega} \\
=e^{t}\left(\tilde{u}_{N}(t)-e^{-|x|^{2}}\left(\tilde{u}_{N}^{2}(t)+2 u_{N}(t) \tilde{u}_{N}(t)\right), \phi\right)_{\omega, N} \\
\quad+(\tilde{f}(t), \phi)_{\omega, N}, \quad \forall \phi \in \mathcal{P}_{N}, 0<t \leq \ln (1+T), \\
\tilde{u}_{N}(0)=\tilde{u}_{N, 0} .
\end{array}\right.
$$

Comparing (4.2) with (3.7), we only have to estimate the upper-bounds of the following terms with $\phi=\tilde{u}_{N}$,

$$
\begin{gathered}
F_{1}(t, \phi)=(\tilde{u}(t), \phi)_{\omega, N}, \quad F_{2}(t, \phi)=\left(e^{-|x|^{2}} \tilde{u}_{N}^{2}(t), \phi\right)_{\omega, N}, \\
F_{3}(t, \phi)=\left(e^{-|x|^{2}} u_{N}(t) \tilde{u}_{N}(t), \phi\right)_{\omega, N}, \quad F_{4}(t, \phi)=(\tilde{f}(t), \phi)_{\omega, N} .
\end{gathered}
$$

By the Schwartz inequality, (2.5) and Lemma 4.1, we have that

$$
\begin{aligned}
& F_{1}\left(t, \tilde{u}_{N}(t)\right)=\left\|\tilde{u}_{N}(t)\right\|_{\omega}^{2}, \\
& \left|F_{2}\left(t, \tilde{u}_{N}(t)\right)\right| \leq\left\|e^{-|x|^{2}} \tilde{u}_{N}^{2}(t)\right\|_{\omega, N}\left\|\tilde{u}_{N}(t)\right\|_{\omega, N} \leq 2 \sqrt{2}\left\|\tilde{u}_{N}(t)\right\|_{\omega}^{2}\left\|\tilde{u}_{N}(t)\right\|_{1, \omega} \\
& \leq 2 \sqrt{2}\left\|\tilde{u}_{N}(t)\right\|_{\omega}\left\|\tilde{u}_{N}(t)\right\|_{1, \omega}^{2} \text {, } \\
& \left|F_{3}\left(t, \tilde{u}_{N}(t)\right)\right|=\left|\left(e^{-|x|^{2}} \tilde{u}_{N}^{2}(t), u_{N}(t)\right)_{\omega, N}\right| \leq\left\|e^{-|x|^{2}} \tilde{u}_{N}^{2}(t)\right\|_{\omega, N}\left\|u_{N}(t)\right\|_{\omega, N} \\
& \leq 2 \sqrt{2}\left\|u_{N}(t)\right\|_{\omega}\left\|\tilde{u}_{N}(t)\right\|_{\omega}\left\|\tilde{u}_{N}(t)\right\|_{1, \omega} \\
& \leq \frac{e^{-t}}{16}\left\|\tilde{u}_{N}(t)\right\|_{1, \omega}^{2}+32 e^{t}\left\|u_{N}(t)\right\|_{\omega}^{2}\left\|\tilde{u}_{N}(t)\right\|_{\omega}^{2}, \\
& \left|F_{4}\left(t, \tilde{u}_{N}(t)\right)\right| \leq\|\tilde{f}(t)\|_{\omega, N}^{2}+\frac{1}{4}\left\|\tilde{u}_{N}(t)\right\|_{\omega}^{2} .
\end{aligned}
$$

Let

$$
\rho\left(\tilde{u}_{N, 0}, \tilde{f}, t\right)=\left\|\tilde{u}_{N, 0}\right\|_{\omega, N}^{2}+2 \int_{0}^{t}\|\tilde{f}(\eta)\|_{\omega, N}^{2} d \eta .
$$

Then the following result follows. 
Theorem 4.1. Let $0 \leq a<1$ and $u_{N}$ be the solution of (4.1). If for certain $t_{1}>0$,

$$
\rho\left(\tilde{u}_{N, 0}, \tilde{f}_{0}, t_{1}\right) \leq \frac{(1-a)^{2}}{16 c_{1}^{2}(T)} e^{-c_{2}\left(u_{N}, T\right) t_{1}},
$$

then for all $t \leq t_{1}$,

$$
\left\|\tilde{u}_{N}(t)\right\|_{\omega}^{2}+\frac{a}{4} \int_{0}^{t}\left\|\tilde{u}_{N}(\eta)\right\|_{1, \omega}^{2} d \eta \leq \rho\left(\tilde{u}_{N, 0}, \tilde{f}_{0}, t\right) e^{c_{2}\left(u_{N}, T\right) t}
$$

where $c_{1}(T)$ is the same as in Theorem 3.1.

Next, we deal with the convergence of scheme (4.1). Let $U_{N}=P_{N} U=P_{N}^{1} U$. We get from (3.5) that

$$
\begin{aligned}
& \left(\partial_{t} U_{N}(t), \phi\right)_{\omega}+\frac{1}{2}\left(U_{N}(t), \phi\right)_{\omega}+\frac{1}{4}\left(\nabla U_{N}(t), \nabla \phi\right)_{\omega} \\
& =e^{t}\left(U_{N}(t)-e^{-|x|^{2}} U_{N}^{2}(t), \phi\right)_{\omega, N}+\sum_{i=1}^{3} G_{i}(t, \phi)+(f(t), \phi)_{\omega, N}, \\
& \quad \forall \phi \in \mathcal{P}_{N}, 0<t \leq \ln (1+T),
\end{aligned}
$$

where

$$
\begin{gathered}
G_{1}(t, \phi)=\left(\partial_{t} U_{N}(t)-\partial_{t} U(t), \phi\right)_{\omega}, \\
G_{2}(t, \phi)=e^{t}\left(e^{-|x|^{2}}\left(U_{N}^{2}(t), \phi\right)_{\omega, N}-e^{t}\left(e^{-|x|^{2}} U^{2}(t), \phi\right)_{\omega},\right. \\
G_{3}(t, \phi)=(f(t), \phi)_{\omega}-(f(t), \phi)_{\omega, N} .
\end{gathered}
$$

Further, let $\tilde{U}_{N}(t)=u_{N}(t)-U_{N}(t)$. Then by (4.1) and (4.3),

$$
\left\{\begin{array}{l}
\left(\partial_{t} \tilde{U}_{N}(t), \phi\right)_{\omega}+\frac{1}{2}\left(\tilde{U}_{N}(t), \phi\right)_{\omega}+\frac{1}{4}\left(\nabla \tilde{U}_{N}(t), \nabla \phi\right)_{\omega} \\
=e^{t}\left(\tilde{U}_{N}(t)-e^{-|x|^{2}}\left(\tilde{U}_{N}^{2}(t)+2 U_{N}(t) \tilde{U}_{N}(t)\right), \phi\right)_{\omega, N} \\
\quad-\sum_{i=1}^{3} G_{i}(t, \phi), \quad \forall \phi \in \mathcal{P}_{N}, 0<t \leq \ln (1+T), \\
\tilde{U}_{N}(0)=I_{N} U_{0}-P_{N} U_{0} .
\end{array}\right.
$$

Comparing (4.4) to (4.2), we only need to estimate $\left|G_{i}\left(t, \tilde{U}_{N}(t)\right)\right|$. Firstly, by Theorem 2.1,

$$
\left|G_{1}\left(t, \tilde{U}_{N}(t)\right)\right| \leq\left\|\tilde{U}_{N}(t)\right\|_{\omega}^{2}+c N^{-r}\left\|\partial_{t} U(t)\right\|_{r, \omega}^{2} .
$$


Next, let

$$
G_{2}\left(t, \tilde{U}_{N}(t)\right)=B_{1}\left(t, \tilde{U}_{N}(t)\right)+B_{2}\left(t, \tilde{U}_{N}(t)\right)
$$

where

$$
\begin{gathered}
B_{1}\left(t, \tilde{U}_{N}(t)\right)=e^{t}\left(e^{-|x|^{2}}\left(U_{N}^{2}(t)-U^{2}(t)\right), \tilde{U}_{N}(t)\right)_{\omega, N}, \\
B_{2}\left(t, \tilde{U}_{N}(t)\right)=e^{t}\left(e^{-|x|^{2}} U^{2}(t), \tilde{U}_{N}(t)\right)_{\omega, N}-e^{t}\left(e^{-|x|^{2}} U^{2}(t), \tilde{U}_{N}(t)\right)_{\omega} .
\end{gathered}
$$

By Lemma 2.3 and Theorem 2.1,

$$
\begin{aligned}
\left\|e^{-\frac{|x|^{2}}{2}}\left(U(t)+U_{N}(t)\right)\right\|_{L^{\infty}} & \leq c\left\|U(t)+U_{N}(t)\right\|_{\omega}^{\frac{1}{2}}\left\|U(t)+U_{N}(t)\right\|_{2, \omega}^{\frac{1}{2}} \\
& \leq c\|U(t)\|_{\omega}^{\frac{1}{2}}\|U(t)\|_{2, \omega}^{\frac{1}{2}} .
\end{aligned}
$$

Thus by (2.5), we have that for $r \geq 2$,

$$
\begin{aligned}
\left|B_{1}\left(t, \tilde{U}_{N}(t)\right)\right| & \leq e^{t}\left\|e^{-\frac{|x|^{2}}{2}}\left(U(t)+U_{N}(t)\right)\right\|_{L^{\infty}}\left\|U(t)-U_{N}(t)\right\|_{\omega, N}\left\|\tilde{U}_{N}(t)\right\|_{\omega, N} \\
& \leq c e^{t}\|U(t)\|_{\omega}^{\frac{1}{2}}\|U(t)\|_{2, \omega}^{\frac{1}{2}}\left\|U(t)-U_{N}(t)\right\|_{\omega}\left\|\tilde{U}_{N}(t)\right\|_{\omega} \\
& \leq \frac{1}{2}\left\|\tilde{U}_{N}(t)\right\|_{\omega}^{2}+c(T) N^{-r}\|U(t)\|_{r, \omega}^{4} .
\end{aligned}
$$

Due to (2.7),

$$
\left|B_{2}\left(t, \tilde{U}_{N}(t)\right)\right| \leq c e^{t} N^{\frac{2}{3}-\frac{r}{2}}\left\|e^{-|x|^{2}} U^{2}(t)\right\|_{r, \omega}\left\|\tilde{U}_{N}(t)\right\|_{\omega} .
$$

It is easy to see that

$$
\begin{aligned}
\partial_{x_{i}}^{r}\left(e^{-|x|^{2}} U^{2}(t)\right)= & e^{-|x|^{2}}\left(2 U(t) \partial_{x_{i}}^{r} U(t)+2 r \partial_{x_{i}} U(t) \partial_{x_{i}}^{r-1} U(t)\right. \\
& \left.+\cdots+p_{r}\left(x_{i}\right) U^{2}(t)\right)
\end{aligned}
$$

where $p_{r}\left(x_{i}\right)$ is a polynomial of degree at most $r$. By Lemma 2.3,

$$
\begin{aligned}
\left\|e^{-|x|^{2}} U(t) \partial_{x_{i}}^{r} U(t)\right\|_{\omega} & \leq\left\|e^{-\frac{|x|^{2}}{2}} U(t)\right\|_{L^{\infty}}\left\|\partial_{x_{i}}^{r} U(t)\right\|_{\omega} \\
& \leq c\|U(t)\|_{r, \omega}^{2}, \\
\left\|e^{-|x|^{2}} p_{r}\left(x_{i}\right) U^{2}(t)\right\|_{\omega} & \leq\left\|e^{-\frac{|x|^{2}}{2}} p_{r}\left(x_{i}\right)\right\|_{L^{\infty}}\left\|e^{-\frac{|x|^{2}}{2}} U(t)\right\|_{L^{\infty}}\|U(t)\|_{\omega} \\
& \leq c\|U(t)\|_{r, \omega}^{2} .
\end{aligned}
$$


By Lemma 3.3 and the Schwartz inequality,

$$
\left\|e^{-|x|^{2}} \partial_{x_{i}} U(t) \partial_{x_{i}}^{r-1} U(t)\right\|_{\omega} \leq c\|U(t)\|_{r, \omega}^{2}, \text { etc.. }
$$

Hence

$$
\left\|e^{-|x|^{2}} U^{2}(t)\right\|_{r, \omega} \leq c\|U(t)\|_{r, \omega}^{2} .
$$

The previous estimates lead to

$$
\left|G_{2}\left(t, \tilde{U}_{N}(t)\right)\right| \leq\left\|\tilde{U}_{N}(t)\right\|_{\omega}^{2}+c(T) N^{\frac{4}{3}-r}\|U(t)\|_{r, \omega}^{4}
$$

where $c(T)$ is a positive constant depending only on $T^{2}$. In addition, Theorem 2.3 implies that for $r \geq 2$,

$$
\begin{aligned}
\left|G_{3}\left(t, \tilde{U}_{N}(t)\right)\right| & \leq c N^{\frac{2}{3}-\frac{r}{2}}\|f(t)\|_{r, \omega}\left\|\tilde{U}_{N}(t)\right\|_{\omega} \\
& \leq\left\|\tilde{U}_{N}(t)\right\|_{\omega}^{2}+\frac{c^{2}}{4} N^{\frac{4}{3}-r}\|f(t)\|_{r, \omega}^{2} .
\end{aligned}
$$

Using Theorem 2.1 and Theorem 2.3,

$$
\left\|\tilde{U}_{N}(0)\right\|_{\omega} \leq c N^{\frac{2}{3}-\frac{r}{2}}\left\|U_{0}\right\|_{r, \omega} .
$$

Finally the following result follows.

Theorem 4.2. If $U \in L^{4}\left(0, \ln (1+T) ; H_{\omega}^{r+\frac{4}{3}}(\Lambda)\right) \cap H^{1}(0, \ln (1+T)$; $\left.H_{\omega}^{r}(\Lambda)\right), f \in L^{2}\left(0, \ln (1+T) ; H_{\omega}^{r+\frac{4}{3}}(\Lambda)\right)$ and $U_{0} \in H_{\omega}^{r+\frac{4}{3}}(\Lambda)$ with $r \geq \frac{2}{3}$, then

$$
\left\|\tilde{U}_{N}(t)\right\|_{\omega}^{2}+\int_{0}^{t}\left\|\tilde{U}_{N}(\eta)\right\|_{1, \omega}^{2} d \eta \leq d^{*} N^{-r}
$$

where $d^{*}$ is a positive constant depending only on $T$ and the norms of $U$ in the spaces mentioned above.

Remark 4.1. By Theorem 4.2 and Theorem 2.1, we have that under the conditions of Theorem 4.2 ,

$$
\left\|u_{N}(t)-U(t)\right\|_{\omega}^{2}+N^{-1} \int_{0}^{t}\left\|u_{N}(\eta)-U(\eta)\right\|_{1, \omega}^{2} d \eta \leq d^{*} N^{-r} .
$$


Remark 4.2. Since $c_{2}\left(U_{N}, T\right)$ depends on $T^{2}$ linearly, we can see that $c^{*}$ depends on $T^{3}$ linearly.

\section{Numerical results}

We present some numerical results in this section. We shall use schemes (3.6) and (4.1) to solve (3.5), respectively. The test function is

$$
U\left(x_{1}, x_{2}, t\right)=\operatorname{sech}^{2}\left(a_{1} x_{1}+a_{2} x_{2}+a_{3} t+a_{4}\right)
$$

with $a_{1}=0.3, a_{2}=0.3, a_{3}=-0.1, a_{4}=3.0$. In actual computation, we use the standard fourth order Runge-Kutta method in time $t$ with the step size $\tau$. The errors of the numerical solution $u_{N}$ are described by

$$
E_{N}(t)=\left\|U(t)-u_{N}(t)\right\|_{\omega, N}, \quad \tilde{E}_{N}(t)=\left\|\frac{U(t)-u_{N}(t)}{U(t)}\right\|_{\omega, N} .
$$

We first use (3.6) to solve (3.5) numerically. The Hermite coefficients are calculated by the Hermite quadratures with $N+1$ interpolation points. The errors $E_{N}(t)$ and $\tilde{E}_{N}(t)$ at $t=1$ with various values of $N$ and $\tau$ are listed in Tables 1 and 2, which show the high accuracy and the convergence of this method. Moreover the errors $E_{N}(t)$ and $\tilde{E}_{N}(t)$ at various time with $N=8$ and $\tau=0.001$ are listed in Table 3, which indicates the stability of calculation. They coincide well with the theoretical analysis in the previous sections.

\begin{tabular}{|c|c|c|c|}
\hline$\tau$ & $N=4$ & $N=8$ & $N=16$ \\
\hline 0.01 & $2.795 \mathrm{E}-03$ & $2.792 \mathrm{E}-04$ & $2.792 \mathrm{E}-04$ \\
\hline 0.001 & $2.824 \mathrm{E}-04$ & $8.793 \mathrm{E}-05$ & $2.983 \mathrm{E}-05$ \\
\hline 0.0001 & $3.278 \mathrm{E}-05$ & $2.801 \mathrm{E}-06$ & $2.799 \mathrm{E}-07$ \\
\hline
\end{tabular}

Table 1 - The errors $E_{N}(1)$.

We next use (4.1) to solve (3.5). The corresponding errors $E_{N}^{(1)}(t)$ and $\tilde{E}_{N}^{(1)}(t)$ are defined in a similar way as for $E_{N}(t)$ and $\tilde{E}_{N}(t)$. The errors $E_{N}^{(1)}(t)$ and $\tilde{E}_{N}^{(1)}(t)$ are presented in Tables 4-6. We find that scheme (3.6) provides the numerical results with the accuracy of the same order as (4.1). But the latter saves the work. Thus it is more preferable in actual computation. 


\begin{tabular}{|c|c|c|c|}
\hline$\tau$ & $N=4$ & $N=8$ & $N=16$ \\
\hline 0.01 & $1.087 \mathrm{E}-01$ & $1.268 \mathrm{E}-02$ & $1.268 \mathrm{E}-02$ \\
\hline 0.001 & $1.085 \mathrm{E}-02$ & $4.070 \mathrm{E}-03$ & $1.670 \mathrm{E}-03$ \\
\hline 0.0001 & $1.929 \mathrm{E}-03$ & $1.070 \mathrm{E}-04$ & $1.065 \mathrm{E}-05$ \\
\hline
\end{tabular}

Table 2 - The errors $\tilde{E}_{N}(1)$.

\begin{tabular}{|c|c|c|}
\hline$t$ & $E_{N}(t)$ & $\tilde{E}_{N}(t)$ \\
\hline 1 & $8.793 \mathrm{E}-05$ & $4.070 \mathrm{E}-03$ \\
\hline 2 & $8.904 \mathrm{E}-05$ & $4.152 \mathrm{E}-03$ \\
\hline 3 & $9.280 \mathrm{E}-05$ & $4.873 \mathrm{E}-03$ \\
\hline 4 & $9.642 \mathrm{E}-05$ & $5.691 \mathrm{E}-03$ \\
\hline 5 & $1.072 \mathrm{E}-04$ & $5.938 \mathrm{E}-03$ \\
\hline
\end{tabular}

Table 3 - The errors $E_{N}(t)$ and $\tilde{E}_{N}(t)$.

As an another example, we take the test function

$$
U\left(x_{1}, x_{2}, t\right)=\frac{\sin \left(b_{1} x_{1}+b_{2} x_{2}\right)}{\left(x_{1}^{2}+x_{2}^{2}+t+1.0\right)^{h}}
$$

with $b_{1}=b_{2}=0.2$ and $h=2.0$. It decays algebraically and oscillates as $x_{1}$ and $x_{2}$ tend to the infinity. We also use (3.6) and (4.1) to solve (3.5) numerically as before. The corresponding errors $E_{N}^{(1)}(t)$ and $\tilde{E}_{N}^{(1)}(t)$ with various $N$ and $t$ are presented in Tables 7-9 for (3.6) and Tables 10-12 for (4.1). They also demonstrate the high accuracy, the covergence and the stability of both schemes.

\begin{tabular}{|c|c|c|c|}
\hline$\tau$ & $N=4$ & $N=8$ & $N=16$ \\
\hline 0.01 & $3.026 \mathrm{E}-03$ & $2.074 \mathrm{E}-04$ & $2.074 \mathrm{E}-04$ \\
\hline 0.001 & $4.799 \mathrm{E}-04$ & $6.562 \mathrm{E}-05$ & $3.862 \mathrm{E}-05$ \\
\hline 0.0001 & $5.469 \mathrm{E}-05$ & $3.009 \mathrm{E}-06$ & $2.724 \mathrm{E}-07$ \\
\hline
\end{tabular}

Table 4 - The errors $E_{N}^{(1)}(1)$. 


\begin{tabular}{|c|c|c|c|}
\hline$\tau$ & $N=4$ & $N=8$ & $N=16$ \\
\hline 0.01 & $1.026 \mathrm{E}-01$ & $1.598 \mathrm{E}-02$ & $1.598 \mathrm{E}-02$ \\
\hline 0.001 & $2.698 \mathrm{E}-02$ & $3.064 \mathrm{E}-03$ & $1.315 \mathrm{E}-03$ \\
\hline 0.0001 & $2.835 \mathrm{E}-03$ & $1.787 \mathrm{E}-04$ & $1.869 \mathrm{E}-05$ \\
\hline
\end{tabular}

Table 5 - The errors $\tilde{E}_{N}^{(1)}(1)$.

\begin{tabular}{|c|c|c|}
\hline$t$ & $E_{N}(t)$ & $\tilde{E}_{N}(t)$ \\
\hline 1 & $6.562 \mathrm{E}-05$ & $3.064 \mathrm{E}-03$ \\
\hline 2 & $6.893 \mathrm{E}-05$ & $3.863 \mathrm{E}-03$ \\
\hline 3 & $7.038 \mathrm{E}-05$ & $4.801 \mathrm{E}-03$ \\
\hline 4 & $8.134 \mathrm{E}-05$ & $6.071 \mathrm{E}-03$ \\
\hline 5 & $9.841 \mathrm{E}-05$ & $8.598 \mathrm{E}-03$ \\
\hline
\end{tabular}

Table 6 - The errors $E_{N}^{(1)}(t)$ and $\tilde{E}_{N}^{(1)}(t)$.

\begin{tabular}{|c|c|c|c|}
\hline$\tau$ & $N=4$ & $N=8$ & $N=16$ \\
\hline 0.01 & $2.440 \mathrm{E}-01$ & $1.438 \mathrm{E}-02$ & $1.438 \mathrm{E}-02$ \\
\hline 0.001 & $4.139 \mathrm{E}-02$ & $5.086 \mathrm{E}-03$ & $1.187 \mathrm{E}-03$ \\
\hline 0.0001 & $8.560 \mathrm{E}-03$ & $5.883 \mathrm{E}-04$ & $3.014 \mathrm{E}-05$ \\
\hline
\end{tabular}

Table 7 - The errors $E_{N}(1)$.

\begin{tabular}{|c|c|c|c|}
\hline$\tau$ & $N=4$ & $N=8$ & $N=16$ \\
\hline 0.01 & $2.484 \mathrm{E}-01$ & $2.431 \mathrm{E}-02$ & $2.431 \mathrm{E}-02$ \\
\hline 0.001 & $4.425 \mathrm{E}-02$ & $4.117 \mathrm{E}-03$ & $2.047 \mathrm{E}-03$ \\
\hline 0.0001 & $6.475 \mathrm{E}-03$ & $4.014 \mathrm{E}-04$ & $2.858 \mathrm{E}-05$ \\
\hline
\end{tabular}

Table 8 - The errors $\tilde{E}_{N}(1)$.

\section{Acknowledgment}

The work of the first author was supported by The Science Foundation of Tongji university of China. The work of the second author was partially supported by The Special Funds for State Major Basic Research Subjects of China N. 


\begin{tabular}{|c|c|c|}
\hline$t$ & $E_{N}(t)$ & $\tilde{E}_{N}(t)$ \\
\hline 1 & $5.086 \mathrm{E}-03$ & $4.117 \mathrm{E}-03$ \\
\hline 2 & $5.323 \mathrm{E}-03$ & $5.135 \mathrm{E}-03$ \\
\hline 3 & $5.855 \mathrm{E}-03$ & $6.087 \mathrm{E}-03$ \\
\hline 4 & $6.957 \mathrm{E}-03$ & $6.087 \mathrm{E}-03$ \\
\hline 5 & $7.366 \mathrm{E}-03$ & $7.416 \mathrm{E}-03$ \\
\hline
\end{tabular}

Table 9 - The errors $E_{N}(t)$ and $\tilde{E}_{N}(t)$.

\begin{tabular}{|c|c|c|c|}
\hline$\tau$ & $N=4$ & $N=8$ & $N=16$ \\
\hline 0.01 & $2.143 \mathrm{E}-01$ & $1.481 \mathrm{E}-02$ & $1.481 \mathrm{E}-02$ \\
\hline 0.001 & $5.107 \mathrm{E}-02$ & $5.187 \mathrm{E}-03$ & $1.119 \mathrm{E}-03$ \\
\hline 0.0001 & $4.480 \mathrm{E}-03$ & $5.734 \mathrm{E}-04$ & $5.884 \mathrm{E}-05$ \\
\hline
\end{tabular}

Table $10-$ The errors $E_{N}^{(1)}(1)$.

\begin{tabular}{|c|c|c|c|}
\hline$\tau$ & $N=4$ & $N=8$ & $N=16$ \\
\hline 0.01 & $2.471 \mathrm{E}-01$ & $1.143 \mathrm{E}-02$ & $1.143 \mathrm{E}-02$ \\
\hline 0.001 & $5.848 \mathrm{E}-02$ & $5.047 \mathrm{E}-03$ & $2.047 \mathrm{E}-03$ \\
\hline 0.0001 & $5.277 \mathrm{E}-03$ & $6.891 \mathrm{E}-04$ & $5.015 \mathrm{E}-05$ \\
\hline
\end{tabular}

Table $11-$ The errors $\tilde{E}_{N}^{(1)}(1)$.

\begin{tabular}{|c|c|c|}
\hline$t$ & $E_{N}(t)$ & $\tilde{E}_{N}(t)$ \\
\hline 1 & $5.187 \mathrm{E}-03$ & $5.047 \mathrm{E}-03$ \\
\hline 2 & $6.114 \mathrm{E}-03$ & $5.863 \mathrm{E}-03$ \\
\hline 3 & $6.082 \mathrm{E}-03$ & $6.854 \mathrm{E}-03$ \\
\hline 4 & $7.571 \mathrm{E}-03$ & $6.976 \mathrm{E}-03$ \\
\hline 5 & $7.841 \mathrm{E}-03$ & $7.445 \mathrm{E}-03$ \\
\hline
\end{tabular}

Table 12 - The errors $E_{N}^{(1)}(t)$ and $\tilde{E}_{N}^{(1)}(t)$.

G1999032804, Shanghai Science Foundation N. 00JC14057 and The Special Funds for Major Speciality of Shanghai Education Committee. 


\section{REFERENCES}

[1] D. Gottlieb and S.A. Orszag, Numerical Analysis of Spectral Methods: Theory and Applications, SIAM-CBMS, Philadelphia, (1977).

[2] Y. Maday, B. Pernaud-Thomas and H. Vandeven, One réhabilitation des méthods spectrales de type Laguerre, Rech. Aérospat., 6 (1985), 353-379.

[3]O. Coulaud, D. Funaro and O. Kavian, Laguerre spectral approximation of elliptic problems in exterior domains, Comp. Mech. in Appl. Mech and Engi., 80 (1990), 451-458.

[4] D. Funaro, Estimates of Laguerre spectral projectors in Sobolev spaces, in Orthogonal Polynomials and Their Applications, ed. by C. Brezinski, L. Gori and A. Ronveaux, Scientific Publishing Co., (1991), 263-266.

[5] Guo Ben-yu and Jie Shen, Laguerre-Galerkin method for nonlinear partial differential equations on a semi-infinite interval, Numer. Math., 86 (2000), 635-654.

[6] D. Funaro and O. Kavian, Approximation of some diffusion evolution equations in unbounded domains by Hermite functions, Math. Comp., 57 (1990), 597-619.

[7] Guo Ben-yu, Error estimation for Hermite spectral method for nonlinear partial differential equations, Math. Comp., 68 (1999), 1067-1078.

[8] Guo Ben-yu and Xu Cheng-long, Hermite pseudospectral method for nonlinear partial differential equations, RAIRO Math. Mdel. and Numer. Anal., 34 (2000), 859-872.

[9] R.A. Adams, Sobolev Spaces, Academic Press, New York, (1975).

[10] Guo Ben-yu, Spectral Methods and Their Applications, World Scientific, Singapore, (1998).

[11] Guo Ben-yu, A class of difference schemes of two-dimensional viscous fluid flow, TR. SUST, 1965, Also see Acta Math. Sinica, 17 (1974), 242-258.

[12] Guo Ben-yu, Generalized stability of discretization and its applications to numerical solution of nonlinear differential equations, Contemporary Math., 163 (1994), 33-54.

[13] H.J. Stetter, Stability of nonlinear discretization algorithms, in Numerical Solutions of Partial Differential Equations, 111-123, ed. by J. Bramble, Academic Press, New York, (1966). 